\title{
Prognostic value of procalcitonin in respiratory tract infections across clinical settings
}

\author{
Alexander Kutz ${ }^{1}$, Matthias Briel ${ }^{2}$, Mirjam Christ-Crain ${ }^{3}$, Daiana Stolz ${ }^{4}$, Lila Bouadma ${ }^{5}$, Michel Wolff ${ }^{5}$, \\ Kristina B Kristoffersen ${ }^{6}$, Long Wei ${ }^{7}$, Olaf Burkhardt ${ }^{8}$, Tobias Welte ${ }^{8}$, Stefan Schroeder ${ }^{9}$, Vandack Nobre ${ }^{10}$, \\ Michael Tamm ${ }^{4}$, Neera Bhatnagar ${ }^{11}$, Heiner C Bucher ${ }^{2}$, Charles-Edouard Luyt ${ }^{12}$, Jean Chastre ${ }^{12}$, Florence Tubach ${ }^{13}$, \\ Beat Mueller ${ }^{1}$ and Philipp Schuetz ${ }^{1 *}$
}

\begin{abstract}
Introduction: Whether the inflammatory biomarker procalcitonin provides prognostic information across clinical settings and different acute respiratory tract infections (ARIs) is poorly understood. In the present study, we investigated the prognostic value of admission procalcitonin levels to predict adverse clinical outcome in a large ARI population.

Methods: We analysed data from 14 trials and 4,211 ARI patients to study associations of admission procalcitonin levels and setting specific treatment failure and mortality alone at 30 days. We used multivariable hierarchical logistic regression and conducted sensitivity analyses stratified by clinical settings and ARI diagnoses to assess the results' consistency.

Results: Overall, 864 patients (20.5\%) experienced treatment failure and 252 (6.0\%) died. The ability of procalcitonin to differentiate patients with from those without treatment failure was highest in the emergency department setting (treatment failure area under the curve (AUC): 0.64 (95\% confidence interval (Cl): 0.61, 0.67), adjusted odds ratio (OR): 1.85 (95\% Cl: 1.61, 2.12), P <0.001; and mortality AUC: 0.67 (95\% Cl: 0.63, 0.71), adjusted OR: 1.82 (95\% Cl: $1.45,2.29), P<0.001)$. In lower respiratory tract infections, procalcitonin was a good predictor of identifying patients at risk for mortality (AUC: 0.71 (95\% Cl: 0.68, 0.74), adjusted OR: 2.13 (95\% Cl: 1.82, 2.49), P <0.001). In primary care and intensive care unit patients, no significant association of initial procalcitonin levels and outcome was found.
\end{abstract}

Conclusions: Admission procalcitonin levels are associated with setting specific treatment failure and provide the most prognostic information regarding ARI in the emergency department setting.

\section{Introduction}

The assessment of disease severity and prediction of adverse outcome in patients with acute respiratory tract infections (ARIs) is essential to improve patient management, including therapeutic and diagnostic steps and site-of-care decisions [1-3]. For this purpose, different blood biomarkers have been evaluated to predict mortality in smaller patient cohorts and critically ill patients, but results are still somewhat controversial in regard to type of biomarker for specific ARI subpopulation and optimal cutoff levels for clinical routine [4-10].

\footnotetext{
* Correspondence: schuetzph@gmail.com

${ }^{1}$ University Department of Medicine, Kantonsspital Aarau, Tellstrasse, 5001 Aarau, Switzerland

Full list of author information is available at the end of the article
}

Procalcitonin (PCT), a precursor protein of calcitonin, is currently one of the most frequently used infectious disease biomarkers in clinical practice [11]. PCT levels mirror severity and evolution of infection and are thought to be associated with poor prognosis in patients with sepsis and ARI [12]. Changes in PCT level in response to therapeutic treatment have also been reported, which suggests prognostic significance in a variety of clinical settings $[13,14]$. However, there is a lack of clinical data comparing PCT in different clinical settings (primary care, emergency department (ED), intensive care unit (ICU)) and across ARI diagnoses to aid in better understanding its (prognostic) value in daily clinical practice.

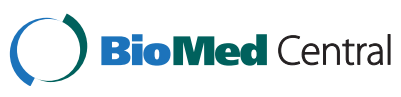

(c) 2015 Kutz et al.; licensee BioMed Central. This is an Open Access article distributed under the terms of the Creative Commons Attribution License (http://creativecommons.org/licenses/by/4.0), which permits unrestricted use, distribution, and reproduction in any medium, provided the original work is properly credited. The Creative Commons Public Domain Dedication waiver (http://creativecommons.org/publicdomain/zero/1.0/) applies to the data made available in this article, unless otherwise stated. 
In the present study, we investigated the prognostic value of admission PCT levels to predict treatment failure and mortality alone in a large ARI patient population across different settings, ARI diagnoses and countries by performing an individual patient data meta-analysis.

\section{Material and methods}

\section{Patients and setting}

This analysis includes all patients from a previous individual patient data meta-analysis [15]. The initial metaanalysis was prespecified in collaboration with the Cochrane Database of Systematic Reviews [16]. In brief, the aim of the meta-analysis was to assess the safety and efficacy of using PCT to guide initiation and duration of antibiotic treatment in patients with ARI assigned to routine PCT measurement or standard of care without PCT measurement. This approach was used over a large range of patients with varying severities of disease in different clinical settings. Patients with a clinical diagnosis of either upper or lower ARI derived from 14 randomised or quasi-randomised trials were included. Trials focused exclusively on paediatric patients or on a purpose other than initiation and duration of antibiotic therapy were not included. Further details about identifying suitable trials were published previously [16]. No ethical approval was needed for this meta-analysis. Written informed consent was obtained from all participants within the initial trials, including consent to participate in further analyses.

\section{Search for and identification of trials}

The initial search was prespecified and published previously [16]. In brief, suitable trials were identified by a formal search of the Cochrane Controlled Trials Registry (CCTR), MEDLINE and Embase (all from their inception to May 2011), as well as reference lists of reports describing such trials. In addition, conference proceedings (from 2006 to 2011) of the Interscience Conference on Antimicrobial Agents and Chemotherapy, the European Congress of Clinical Microbiology and Infectious Disease, the American Thoracic Society, the American Association of Respiratory Care and the American College of Chest Physicians were hand-searched. Trial registries were checked and experts contacted for further eligible trials. Two independent reviewers assessed trial eligibility on basis of titles, abstracts, full-text reports and further information obtained from investigators as needed. Further, the protocol, case report forms and unedited databases from investigators of all eligible trials were requested. Data from each trial were first checked against reported results, and queries were resolved with the principal investigator, trial data manager or statistician.

\section{Aims and endpoints}

The aim of the present analysis was to study associations of admission PCT levels and adverse clinical outcomes. In line with the initial Cochrane meta-analysis protocol [16], the predefined primary endpoints were defined as all-cause mortality and setting specific treatment failure at 30 days. For the primary care setting, treatment failure was defined as occurrence of at least one of the following events: death, hospitalisation, ARI-specific complications (for example, empyema for lower ARI, meningitis for upper ARI), recurrent or worsening infection, and patients reporting any symptoms of an ongoing respiratory infection (for example, fever, cough, dyspnoea) at 30-day follow-up. For the ED setting, treatment failure was defined as death, ICU admission, rehospitalisation after index hospital discharge, ARI-associated complications (for example, empyema or acute respiratory distress syndrome for lower ARI), or recurrent or worsening infection within 30 days of follow-up. For the ICU setting, treatment failure was defined as death within 30 days of follow-up.

In regard to PCT as a baseline predictor, we used initial PCT levels corresponding to the PCT level drawn at the primary care visit (primary care), ED admission (ED trials) and ICU admission (ICU trials). In all trials, PCT was measured using a rapid, sensitive assay with a functional assay sensitivity of $0.06 \mu \mathrm{g} / \mathrm{L}$ (KRYPTOR PCT; $\mathrm{B} \cdot \mathrm{R} \cdot \mathrm{A} \cdot \mathrm{H} \cdot \mathrm{M} \cdot \mathrm{S}$, Hennigsdorf, Germany) and an assay time of less than 20 minutes. We used different a priori defined PCT cutoffs $(0.1 \mu \mathrm{g} / \mathrm{L}, 0.25 \mu \mathrm{g} / \mathrm{L}, 0.5 \mu \mathrm{g} / \mathrm{L}, 2.0 \mu \mathrm{g} / \mathrm{L})$ that correspond to cutoffs used in previous antibiotic stewardship trials and also in practice guidelines on the use of PCT.

\section{Statistical analysis}

We used descriptive statistics, including mean with standard deviation, median with interquartile range (IQR) and frequencies, to describe the populations, as appropriate.

Patients were divided in two groups depending on having experienced a treatment failure or not. Because the rate of loss to follow-up in the different trials was low $(<10 \%)$, we assumed that patients lost to follow-up did not undergo an event. This assumption was also verified in a time-to-event analysis where we used censoring for patients lost to follow-up.

For the primary endpoint of setting- and diagnosisspecific treatment failure or all-cause mortality alone, we calculated adjusted odds ratios (ORs) (with age and sex as additional fixed effects) and 95\% confidence intervals (CIs) using multivariable hierarchical logistic regression. Discrimination of PCT levels for treatment failure or mortality was investigated by area under the receiver operating characteristic (ROC) curve and 95\% CIs. For the different clinical settings (primary care, ED, ICU) and most prevalent ARI 
subgroups (that is, acute bronchitis, exacerbated chronic obstructive pulmonary disease (ECOPD), community acquired pneumonia (CAP)), we calculated sensitivity and specificity analyses. We used different clinically established PCT cutoffs $(0.1 \mu \mathrm{g} / \mathrm{L}, 0.25 \mu \mathrm{g} / \mathrm{L}, 0.5 \mu \mathrm{g} / \mathrm{L}, 2.0 \mu \mathrm{g} / \mathrm{L})$ to estimate risk prediction. If subgroups were too small, values were labelled as 'not applicable'. For graphical display, we calculated Kaplan-Meier curves for time to death and time to adverse outcome.

Tests were carried out at $5 \%$ significance levels. Analyses were performed with STATA 12.1 software (StataCorp, College Station, TX, USA).

\section{Results}

\section{Population}

The study sample comprised all 4,211 intention-to-treat patients (median age: 63 years, $54.2 \%$ male) with a final diagnosis of ARI. Regarding the clinical setting, a total of 1,008 (23.9\%) patients were from primary care, 2,605 (61.9\%) from the ED and 598 (14.2\%) from the ICU. Overall, 864 patients (20.5\%) had a treatment failure and $252(6.0 \%)$ patients died. Baseline characteristics of the overall population and stratified based on the occurrence of a treatment failure are summarized in Table 1.

\section{Procalcitonin levels in different settings and acute respiratory tract infections}

As shown in Figure 1A, PCT levels significantly increased with higher-acuity clinical settings (that is, primary care, ED, ICU). Similar results were found when we looked at PCT levels in different types of ARI with higher levels in more severe ARIs (that is, ECOPD, CAP, ventilator-associated pneumonia) (Figure 1B).

\section{Association of admission procalcitonin levels and adverse outcome}

Table 2 summarizes the results of logistic regression analysis as a measure of association and AUC as a measure of discrimination for both endpoints: treatment failure and mortality. PCT levels in ED patients were significantly associated with treatment failure (AUC: 0.64 (95\% CI: 0.61, 0.67), adjusted OR: 1.85 (95\% CI: 1.61, 2.12), $P<0.001$ ) and mortality (AUC: 0.67 (95\% CI: 0.63, 0.71), adjusted OR: 1.82 (95\% CI: 1.45, 2.29), $P<0.001$ ), whereas no significant effect was seen in primary care or ICU patients. In

Table 1 Baseline characteristics ${ }^{a}$

\begin{tabular}{|c|c|c|c|}
\hline Parameter & All $(n=4,211)$ & $\begin{array}{l}\text { Patients with treatment failure } \\
(\mathrm{n}=864)\end{array}$ & $\begin{array}{l}\text { Patients without treatment failure } \\
(\mathrm{n}=3,347)\end{array}$ \\
\hline Age (yr), median (IQR) & 63 (44 to 76$)$ & 63 (45 to 76$)$ & 63 (44 to 76$)$ \\
\hline Men, n (\%) & $2,282(54.2)$ & $438(50.7)$ & $1,844(55.1)$ \\
\hline \multicolumn{4}{|l|}{ Clinical setting, n (\%) } \\
\hline Primary care & $1,008(23.9)$ & $323(37.4)$ & $685(20.5)$ \\
\hline Emergency department & $2,605(61.9)$ & $410(47.5)$ & 2,195 (65.6) \\
\hline ICU & $598(14.2)$ & $131(15.2)$ & $467(14.0)$ \\
\hline \multicolumn{4}{|l|}{ Primary diagnosis } \\
\hline Total upper ARI, n (\%) & $549(13.0)$ & $185(21.4)$ & $364(10.9)$ \\
\hline Common cold & $305(7.2)$ & $105(12.2)$ & $200(6.0)$ \\
\hline Rhinosinusitis, otitis & $137(3.3)$ & $46(5.3)$ & $91(2.7)$ \\
\hline Pharyngitis, tonsillitis & $107(2.5)$ & $34(3.9)$ & $73(2.2)$ \\
\hline Total lower ARI, n (\%) & $3,567(84.7)$ & $654(75.7)$ & $2,913(87.0)$ \\
\hline Community-acquired pneumonia & $2,027(48.1)$ & $430(49.8)$ & $1,597(47.7)$ \\
\hline Hospital-acquired pneumonia & $79(1.9)$ & $0(0)$ & $79(2.4)$ \\
\hline Ventilator-associated pneumonia & $242(5.7)$ & $20(2.3)$ & $222(6.7)$ \\
\hline Acute bronchitis & $531(12.6)$ & $105(12.2)$ & $426(12.7)$ \\
\hline Exacerbation of COPD & $584(13.9)$ & $80(9.3)$ & $504(15.1)$ \\
\hline Exacerbation of asthma & $30(0.7)$ & $5(0.6)$ & $25(0.7)$ \\
\hline Unspecified lower ARI & $74(1.8)$ & $14(1.6)$ & $60(1.8)$ \\
\hline Other final diagnosis, n (\%) & $95(2.3)$ & $25(2.9)$ & $70(2.1)$ \\
\hline $\begin{array}{l}\text { Procalcitonin overall }(\mu \mathrm{g} / \mathrm{L}) \text {, mean, median } \\
(\mathrm{SD}, \mathrm{IQR})\end{array}$ & $2.5,0.2(11.4,0.1$ to 0.8$)$ & $4.4,0.2(18.7,0.1$ to 1.2$)$ & $2.0,0.2(8.4,0.1$ to 0.8$)$ \\
\hline
\end{tabular}

${ }^{\mathrm{a}} \mathrm{ARI}$, Acute respiratory tract infection; COPD, Chronic obstructive pulmonary disease; ICU, Intensive care unit; IQR, Interquartile range; PCT, Procalcitonin; SD, Standard deviation. 


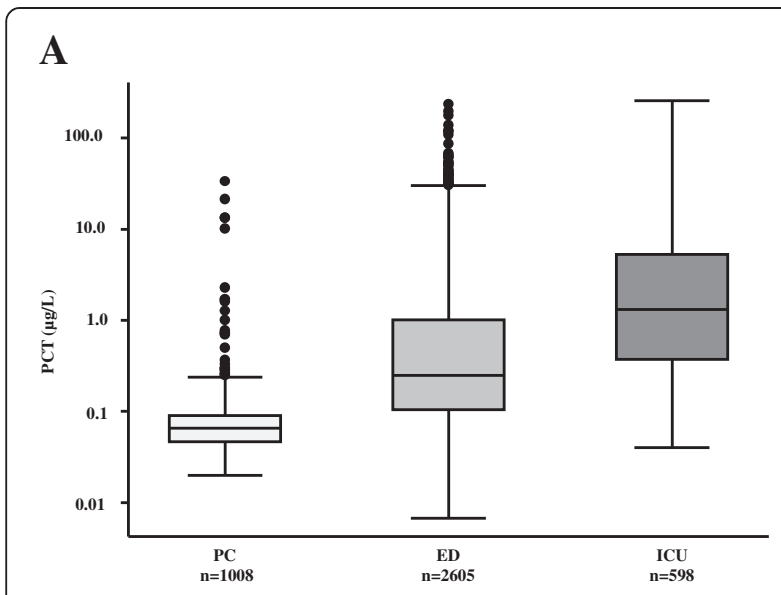

B

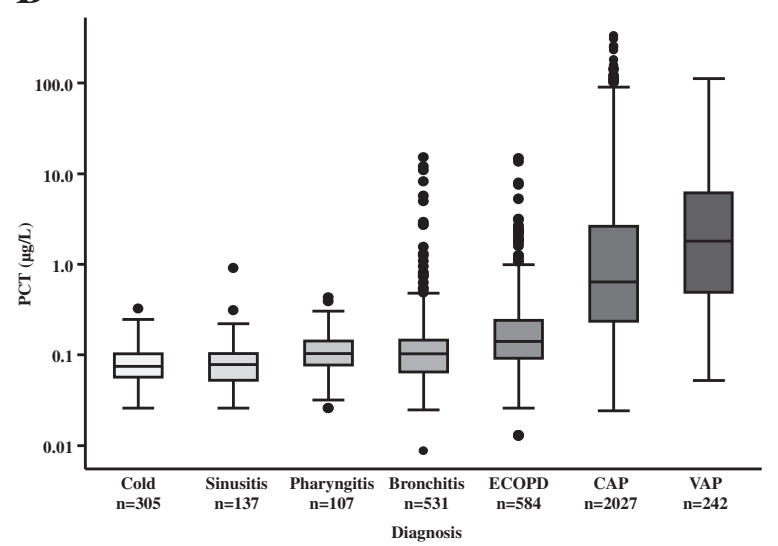

Figure 1 Admission procalcitonin levels. (A) Procalcitonin $(P C T)$ levels stratified by setting $(P=0.0001)$. (B) PCT levels stratified by diagnosis $(P=0.0001)$. ECOPD, Exacerbated chronic obstructive pulmonary disease; ED, Emergency department; ICU, Intensive care unit; CAP, Community-acquired pneumonia; PC, Primary care; VAP, Ventilator-associated pneumonia.

patients with common cold, PCT could significantly predict treatment failure (AUC: 0.58 (95\% CI: 0.51, 0.64), adjusted OR: 4.76 (95\% CI: 1.27, 17.87), $P=0.021)$. Nevertheless, PCT did not deliver supplementary prognostic information in the overall upper ARI population. In lower ARI, PCT was significantly associated with treatment failure (AUC: 0.57 (95\% CI: 0.54, 0.59), adjusted OR: 1.40 (95\% CI: $1.26,1.55), P<0.001$ ) and mortality (AUC: 0.71 (95\% CI: 0.68, 0.74), adjusted OR: 2.13 (95\% CI: 1.82, 2.49), $P<0.001$ ). In patients with acute bronchitis (AUC: 0.91 (95\% CI: 0.88, 0.95), adjusted OR: 1.97 (95\% CI: 0.20, 19.52), $P=0.561$ ) and ECOPD (AUC: 0.79 (95\% CI: 0.69 , 0.90 ), adjusted OR: 6.12 (95\% CI: 2.46, 15.18), $P<0.001$ ), admission PCT levels were good predictors to identify patients at risk for mortality. Significantly different PCT levels in patients with versus without events (treatment failure or mortality) were also observed in ECOPD and CAP, as detailed in Table 2. Figure 2 shows the results of the adjusted regression analysis for treatment failure and mortality in the different populations studied.

PCT in the different cutoff ranges significantly separated ED patients in regard to the time to treatment failure (Figure 3A) and time to mortality (Figure 3B) $(P<0.0001$ and $P=0.02$, respectively). Similar analyses did not show significant results in the primary care and ICU settings (data not shown). In the overall lower ARI population, as well as in CAP patients, PCT also showed significant separation in regard to the time to treatment failure $(P<0.0001$ and $P<0.01$, respectively) and time to mortality $(P<0.0001$ and $P<0.01$, respectively) (Figure 4$)$.

Tables 3 and 4 gives an overview of diagnostic measures (including sensitivity, specificity, negative predictive values (NPVs) and positive predictive values (PPVs)) of PCT at different PCT cutoff levels. In primary care patients, a cutoff of $0.25 \mu \mathrm{g} / \mathrm{L}$ had a high PPV of $45.8 \%$ (95\% CI: $25.6,67.2)$ and a high specificity of $98.1 \%$ (95\% CI: 96.8, 99.0) for treatment failure. At the same cutoff, we found a high NPV of $89.2 \%$ (95\% CI: 87.4, 90.8) and a sensitivity of $65.6 \%$ (95\% CI: $60.8,70.2)$ for treatment failure and a NPV of 97.5\% (95\% CI: 96.5, 98.3) and a sensitivity of $72.5 \%$ (95\% CI: 63.6, 80.3) for mortality in ED patients. Similarly, in patients with lower ARIs, a low PCT cutoff showed high levels of sensitivity, whereas levels of specificity were higher with higher PCT cutoffs. In patients with CAP, the NPV of PCT at the 0.1 and $0.25 \mu \mathrm{g} / \mathrm{L}$ cutoffs were high for treatment failure $(85.5 \%$ (95\% CI: $80.2,89.9)$ and $84.9 \%$ (95\% CI: 81.9, 87.6)) as well as for mortality (95.5\% (95\% CI: 91.8, 97.8) and 94.2\% (95\% CI: 92.2, 95.9)). For ICU patients, diagnostic measures remained somewhat undetermined at all the different PCT cutoffs.

\section{Discussion}

The findings of this analysis, which included a large population with different types and severities of ARI from previous randomised controlled trials, are threefold. First, we found an increase in PCT levels across clinical settings and ARI diagnoses, demonstrating that the current practice of using different cutoff levels in different clinical settings is mandatory. Second, initial PCT levels were significantly associated with setting-specific treatment failure and mortality in the overall population. Third, the prognostic information derived from initial PCT levels in primary care and ICU patients was rather low, whereas in the ED setting and in ECOPD and bronchitis patients, admission PCT levels provided prognostic information and thereby may improve initial risk stratification in these patient populations.

An accurate and fast assessment of disease severity and predictions regarding a patient's clinical course potentially assist patients and physicians with settingappropriate expectations regarding the illness. Such 
Table 2 Procalcitonin levels at day 0 for prediction of severe adverse events in patients with acute respiratory infection ${ }^{a}$

\begin{tabular}{|c|c|c|c|c|c|c|}
\hline & Event & $\begin{array}{l}\mathrm{PCT}(\mu \mathrm{g} / \mathrm{L}) \text { in patients with } \\
\text { treatment failure }\end{array}$ & $\begin{array}{l}\mathrm{PCT}(\mu \mathrm{g} / \mathrm{L}) \text { in patients without } \\
\text { treatment failure }\end{array}$ & $\begin{array}{l}\text { Adjusted OR } \\
(95 \% \mathrm{Cl})\end{array}$ & AUC $(95 \% \mathrm{Cl})$ & $P$-value \\
\hline \multicolumn{7}{|l|}{ Clinical setting, mean, median (SD, IQR) } \\
\hline Primary care & Treatment failure & $0.26,0.07(2.22,0.05$ to 0.09$)$ & $0.14,0.07(0.83,0.05$ to 0.09$)$ & $1.12(0.73,1.72)$ & $0.50(0.46,0.54)$ & 0.606 \\
\hline \multirow[t]{2}{*}{ Emergency department } & Treatment failure & $5.26,0.51(17.86,0.17$ to 2.71$)$ & $1.85,0.23(8.09,0.10$ to 0.80$)$ & $1.85(1.61,2.12)$ & $0.64(0.61,0.67)$ & $<0.001$ \\
\hline & Mortality & $5.85,0.54(22.53,0.21$ to 3.17$)$ & $2.22,0.24(9.33,0.10$ to 0.96$)$ & $1.82(1.45,2.29)$ & $0.67(0.63,0.71)$ & $<0.001$ \\
\hline ICU & Mortality & $12.06,1.15(35.21,0.33$ to 6.34$)$ & $6.59,1.36(14.07,0.37$ to 5.07$)$ & $1.05(0.81,1.37)$ & $0.50(0.44,0.56)$ & 0.705 \\
\hline \multicolumn{7}{|l|}{ Primary diagnosis } \\
\hline Total upper ARI, n (\%) & Treatment failure & $0.07,0.06(0.04,0.05$ to 0.08$)$ & $0.07,0.06(0.05,0.04$ to 0.08$)$ & $0.91(0.40,2.10)$ & $0.50(0.45,0.55)$ & 0.832 \\
\hline Common cold & Treatment failure & $0.07,0.06(0.03,0.05$ to 0.08$)$ & $0.06,0.05(0.03,0.04$ to 0.08$)$ & $4.76(1.27,17.87)$ & $0.58(0.51,0.64)$ & 0.021 \\
\hline Rhinosinusitis, otitis & Treatment failure & $0.06,0.05(0.03,0.03$ to 0.08$)$ & $0.08,0.06(0.08,0.04$ to 0.09$)$ & $0.18(0.03,0.96)$ & $0.39(0.29,0.50)$ & 0.045 \\
\hline Pharyngitis, tonsillitis & Treatment failure & $0.08,0.07(0.05,0.06$ to 0.10$)$ & $0.09,0.08(0.05,0.06$ to 0.11$)$ & $0.19(0.02,1.53)$ & $0.42(0.31,0.54)$ & 0.120 \\
\hline \multirow[t]{2}{*}{ Total lower ARI, n (\%) } & Treatment failure & $5.64,0.38(21.20,0.11$ to 1.91$)$ & $2.34,0.23(8.97,0.09$ to 1.02$)$ & $1.40(1.26,1.55)$ & $0.57(0.54,0.59)$ & $<0.001$ \\
\hline & Mortality & $9.3,0.95(30.25,0.30$ to 5.11$)$ & $2.49,0.22(9.59,0.09$ to 1.04$)$ & $2.13(1.82,2.49)$ & $0.71(0.68,0.74)$ & $<0.001$ \\
\hline \multirow[t]{2}{*}{ Acute bronchitis } & Treatment failure & $0.10,0.07(0.11,0.05$ to 0.11$)$ & $0.22,0.08(0.93,0.05$ to 0.12$)$ & $0.77(0.40,1.48)$ & $0.47(0.41,0.53)$ & 0.430 \\
\hline & Mortality & $0.22,0.22(0.04,0.19$ to 0.24$)$ & $0.20,0.08(0.84,0.05$ to 0.11$)$ & $1.97(0.20,19.52)$ & $0.91(0.88,0.95)$ & 0.561 \\
\hline \multirow[t]{2}{*}{ Exacerbation of COPD } & Treatment failure & $0.40,0.14(1.30,0.08$ to 0.27$)$ & $0.23,0.10(0.67,0.07$ to 0.17$)$ & $1.94(1.14,3.32)$ & $0.60(0.53,0.67)$ & 0.015 \\
\hline & Mortality & $1.06,0.25(2.70,0.18$ to 0.62$)$ & $0.23,0.10(0.64,0.07$ to 0.18$)$ & $6.12(2.46,15.18)$ & $0.79(0.69,0.90)$ & $<0.001$ \\
\hline \multirow[t]{2}{*}{ Community-acquired pneumonia } & Treatment failure & $8.30,1.00(25.76,0.28$ to 5.19$)$ & $3.13,0.41(10.56,0.17$ to 1.57$)$ & $1.66(1.44,1.90)$ & $0.61(0.58,0.64)$ & $<0.001$ \\
\hline & Mortality & $10.66,1.01(32.95,0.33$ to 5.67$)$ & $3.53,0.44(11.63,0.18$ to 1.76$)$ & $1.69(1.41,2.04)$ & $0.62(0.58,0.66)$ & $<0.001$ \\
\hline Ventilator-associated pneumonia & Mortality & $4.27,1.29(6.43,0.39$ to 5.87$)$ & $5.34,1.42(11.81,0.37$ to 4.47$)$ & $1.08(0.55,2.12)$ & $0.51(0.38,0.65)$ & 0.817 \\
\hline
\end{tabular}

${ }^{\mathrm{a} A R I}$, Acute respiratory tract infection; AUC, Area under the curve; $\mathrm{Cl}$, Confidence interval; COPD, Chronic obstructive pulmonary disease; ICU, Intensive care unit; IQR, interquartile range; OR, odds ratio; PCT, procalcitonin; SD, standard deviation. Statistically significant values are shown in bold. 


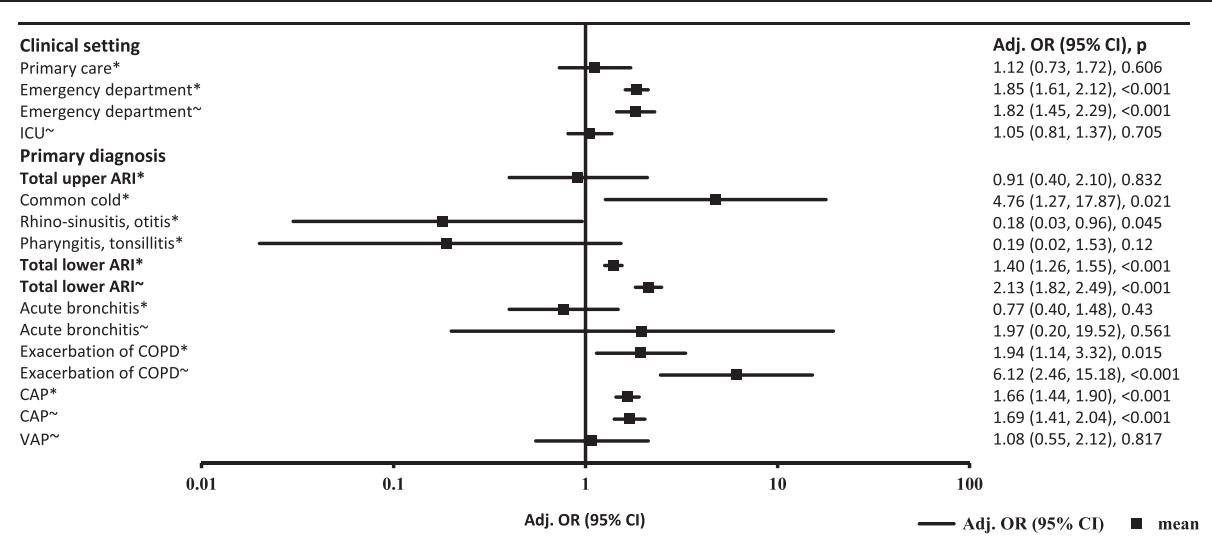

Figure 2 Multivariate regression analysis for estimation of predictive value of procalcitonin levels on admission stratified by adverse events and mortality in different settings and diagnoses. *Treatment failure; Mortality; Adj., Adjusted; ARI, Acute respiratory tract infection; CAP, Community-acquired pneumonia; Cl, Confidence interval; ICU, Intensive care unit; COPD, Chronic obstructive pulmonary disease OR, Odds ratio; VAP, Ventilator-associated pneumonia.
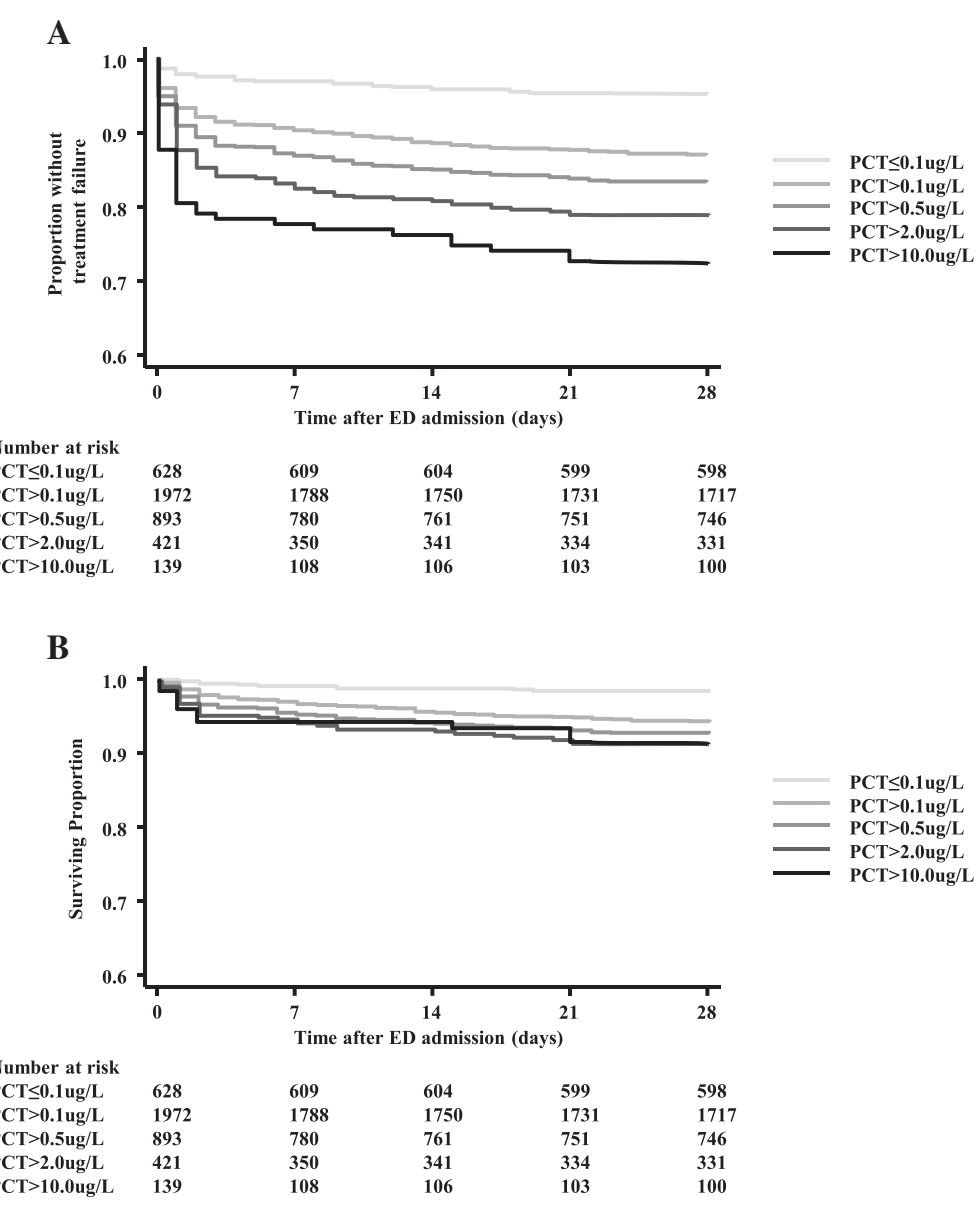

Figure 3 Association between time to severe adverse events and admission procalcitonin levels in emergency department patients. (A) Time to treatment failure is significantly shorter in emergency department (ED) patients with higher procalcitonin (PCT) levels on admission. $(P<0.0001$. (B) Time to death is significantly shorter in ED patients with higher PCT levels on admission $(P<0.02)$. Treatment failure is defined as death, ICU admission, rehospitalisation, complications or recurrent or worsening infection within 28 days. 


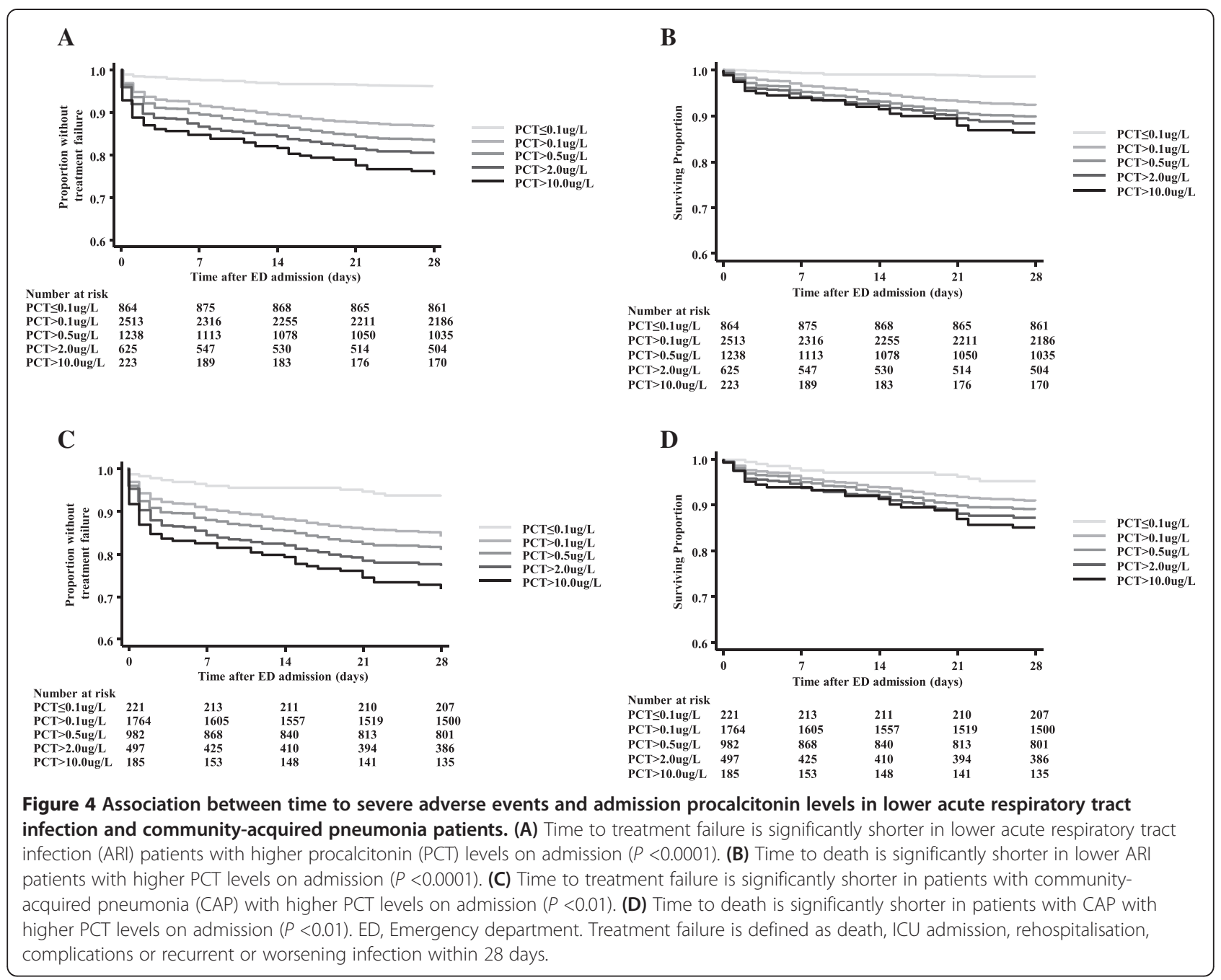

predictions are of particular importance to ensuring efficient use of health care and hospital resources and are indispensable for choosing optimal therapeutic options in the initial management of ARIs [17]. This includes decisions regarding site of care, diagnostic evaluation and assessment for appropriate early discharge [18].

The role of prognostication is also acknowledged by respiratory infection guidelines, which recommend stratifying patients with CAP on the basis of predicted risk for mortality using validated risk scores (that is, the Pneumonia Severity Index (PSI) or CURB-65 score) $[19,20]$. Clinical risk scores are somewhat limited by practicality and risk for miscalibration due to different patient populations, and therefore they have only moderate operational characteristics [21]. Also, these scores are validated only for CAP and not for other ARIs. Thus, there is interest in additional prognosticating mechanisms, throughout all clinical settings and subgroups of ARIs, using newly available biomarkers (such as PCT) that are objectively and rapidly measurable, as well as responsive to clinical recovery, and that add relevant, reliable, real-time information.

The prognostic potential of PCT in patients with respiratory infections has been evaluated in different studies, mainly in patients with CAP and sepsis. Most studies have found PCT levels to be increased in patients not surviving their disease compared with survivors, but with only moderate prognostic accuracy. In line with our results, in a large CAP cohort in the United States [22], the greatest benefit of PCT was found in patients classified as high risk on the basis of PSI score. Having a PCT $<0.1 \mu \mathrm{g} / \mathrm{L}$ virtually excluded mortality in these high-risk patients. In a Swiss study, researchers found that initial PCT levels did not improve clinical risk scores for mortality prediction [23]. Subsequent repeated measurements of PCT in this population demonstrated improved clinical outcomes with falling PCT levels. In addition, the study found that PCT was more helpful in predicting treatment failure other than 


\section{Table 3 Procalcitonin cutoffs ( 0.1 and $0.25 \mu \mathrm{g} / \mathrm{L})$ for risk prediction in different clinical settings and in various acute respiratory infection subgroups ${ }^{\mathrm{a}}$}

\begin{tabular}{|c|c|c|c|c|c|c|c|c|}
\hline \multirow[t]{3}{*}{ Cutoff $(\mu \mathrm{g} / \mathrm{L})$} & \multicolumn{4}{|l|}{$0.1 \mu \mathrm{g} / \mathrm{L}$} & \multicolumn{4}{|l|}{$0.25 \mu \mathrm{g} / \mathrm{L}$} \\
\hline & Sensitivity (\%) & Specificity (\%) & PPV (\%) & NPV (\%) & Sensitivity (\%) & Specificity (\%) & PPV (\%) & NPV (\%) \\
\hline & $(95 \% \mathrm{Cl})$ & $(95 \% \mathrm{Cl})$ & $(95 \% \mathrm{Cl})$ & $(95 \% \mathrm{Cl})$ & $(95 \% \mathrm{Cl})$ & $(95 \% \mathrm{Cl})$ & $(95 \% \mathrm{Cl})$ & $(95 \% \mathrm{Cl})$ \\
\hline \multicolumn{9}{|l|}{ PC } \\
\hline Treatment failure & $19.8(15.6,24.6)$ & $81.3(78.2,84.1)$ & $33.3(26.7,40.5)$ & $68.2(64.9,71.4)$ & $3.41(1.71,6.01)$ & $98.1(96.8,99.0)$ & $45.8(25.6,67.2)$ & $68.3(65.2,71.2)$ \\
\hline \multicolumn{9}{|l|}{ ED } \\
\hline Treatment failure & $86.1(82.4,89.3)$ & $26.1(24.2,28.0)$ & $17.9(16.2,19.7)$ & $90.9(88.4,93.1)$ & $65.6(60.8,70.2)$ & $53.1(51.0,55.2)$ & $20.8(18.6,23.1)$ & $89.2(87.4,90.8)$ \\
\hline Mortality & $92.5(86.2,96.5)$ & $25.0(23.3,26.7)$ & $5.63(4.65,6.74)$ & $98.6(97.3,99.3)$ & $72.5(63.6,80.3)$ & $51.2(49.3,53.2)$ & $6.71(5.4,8.2)$ & $97.5(96.5,98.3)$ \\
\hline \multicolumn{9}{|l|}{ ICU } \\
\hline Mortality & $95.2(89.8,98.2)$ & $4.21(2.43,6.75)$ & $24.6(20.9,28.7)$ & $72.7(49.8,89.3)$ & $80.8(72.8,87.3)$ & $18.9(15.1,23.3)$ & $24.7(20.6,29.2)$ & $75.0(65.1,83.3)$ \\
\hline \multicolumn{9}{|l|}{ Total lower ARI } \\
\hline Treatment failure & $76.4(72.9,79.6)$ & $28.2(26.5,29.9)$ & $19.6(18.1,21.2)$ & $83.9(81.4,86.1)$ & $57.4(53.5,61.3)$ & $52.8(51.0,54.7)$ & $21.8(19.9,23.9)$ & $84.4(82.6,86.0)$ \\
\hline Mortality & $94.5(90.8,97.0)$ & $28.9(27.4,30.5)$ & $8.89(7.8,10.1)$ & $98.6(97.7,99.3)$ & $78.1(72.2,83.2)$ & $53.0(51.3,54.8)$ & $10.9(9.42,12.4)$ & $97.1(96.2,97.8)$ \\
\hline \multicolumn{9}{|l|}{ Bronchitis } \\
\hline Treatment failure & $28.6(20.2,38.2)$ & $68.0(63.3,72.4)$ & $18.1(12.5,24.8)$ & $79.4(74.9,83.4)$ & $3.81(1.05,9.47)$ & $92.5(89.5,94.8)$ & $11.1(3.11,26.1)$ & $79.6(75.7,83.0)$ \\
\hline Mortality & $100(15.8,100)$ & $68.9(64.8,72.9)$ & $1.20(0.15,4.28)$ & $100(99.0,100)$ & $0.00(0.00,84.2)$ & $93.2(90.7,95.2)$ & $0.00(0.00,9.74)$ & $99.6(98.5,100)$ \\
\hline \multicolumn{9}{|l|}{ ECOPD } \\
\hline Treatment failure & $60.0(48.4,70.8)$ & $49.8(45.3,54.3)$ & $16.0(12.0,20.6)$ & $88.7(84.4,92.1)$ & $27.5(18.1,38.6)$ & $85.9(82.5,88.8)$ & $23.7(15.5,33.6)$ & $88.1(84.9,90.9)$ \\
\hline Mortality & $82.4(56.6,96.2)$ & $49.4(45.2,53.6)$ & $4.67(2.57,7.71)$ & $98.9(96.9,99.8)$ & $47.1(23.0,72.2)$ & $85.0(81.7,87.8)$ & $8.60(3.79,16.2)$ & $98.2(96.5,99.2)$ \\
\hline \multicolumn{9}{|l|}{ CAP } \\
\hline Treatment failure & $92.5(89.5,94.8)$ & $12.1(10.5,13.8)$ & $22.2(20.3,24.2)$ & $85.5(80.2,89.9)$ & $77.1(72.8,81.0)$ & $35.0(32.6,37.4)$ & $24.3(22.1,26.7)$ & $84.9(81.9,87.6)$ \\
\hline Mortality & $94.9(90.9,97.5)$ & $11.8(10.3,13.4)$ & $10.6(9.20,12.1)$ & $95.5(91.8,97.8)$ & $81.2(75.1,86.4)$ & $33.9(31.7,36.1)$ & $11.9(10.2,13.8)$ & $94.2(92.2,95.9)$ \\
\hline
\end{tabular}

${ }^{\mathrm{a} A R I}$, Acute respiratory tract infection; CAP, Community-acquired pneumonia; Cl, Confidence interval; ECOPD, Exacerbated chronic obstructive pulmonary disease; ED, Emergency department; ICU, Intensive care unit; NA, Not applicable due to small sample size; NPV, Negative predictive value; PC, Primary care; PCT, Procalcitonin; PPV, Positive predictive value. 
Table 4 Procalcitonin cutoffs $(0.5$ and $2.0 \mu \mathrm{g} / \mathrm{L})$ for risk prediction in different clinical settings and in various acute respiratory infection subgroups ${ }^{\mathrm{a}}$

\begin{tabular}{|c|c|c|c|c|c|c|c|c|}
\hline \multirow[t]{3}{*}{ Cutoff $(\mu \mathrm{g} / \mathrm{L})$} & \multicolumn{4}{|l|}{$0.5 \mu \mathrm{g} / \mathrm{L}$} & \multicolumn{4}{|l|}{$2.0 \mu \mathrm{g} / \mathrm{L}$} \\
\hline & Sensitivity (\%) & Specificity (\%) & PPV (\%) & NPV (\%) & Sensitivity (\%) & Specificity (\%) & PPV (\%) & NPV (\%) \\
\hline & $(95 \% \mathrm{Cl})$ & $(95 \% \mathrm{Cl})$ & $(95 \% \mathrm{Cl})$ & $(95 \% \mathrm{Cl})$ & $(95 \% \mathrm{Cl})$ & $(95 \% \mathrm{Cl})$ & $(95 \% \mathrm{Cl})$ & $(95 \% \mathrm{Cl})$ \\
\hline \multicolumn{9}{|l|}{ PC } \\
\hline Treatment failure & $1.86(0.69,4.00)$ & $98.7(97.5,99.4)$ & $40.0(16.3,67.7)$ & $68.0(65.0,70.9)$ & NA & NA & NA & NA \\
\hline \multicolumn{9}{|l|}{ ED } \\
\hline Treatment failure & $50.2(45.3,55.2)$ & $68.6(66.6,70.6)$ & $23.1(20.3,26.0)$ & $88.0(86.4,89.6)$ & $27.6(23.3,32.2)$ & $85.9(84.4,87.4)$ & $26.8(22.7,31.3)$ & $86.4(84.9,87.8)$ \\
\hline Mortality & $52.5(43.2,61.7)$ & $66.5(64.6,68.4)$ & $7.05(5.46,8.94)$ & $96.7(95.7,97.5)$ & $28.3(20.5,37.3)$ & $84.4(82.9,85.8)$ & $8.08(5.66,11.1)$ & $96.1(95.1,96.8)$ \\
\hline \multicolumn{9}{|l|}{ ICU } \\
\hline Mortality & $67.2(58.2,75.3)$ & $30.8(26.2,35.7)$ & $24.2(19.8,29.1)$ & $74.1(66.5,80.7)$ & $37.6(29.1,46.7)$ & $58.2(53.0,63.2)$ & $22.8(17.3,29.2)$ & $73.9(68.5,78.8)$ \\
\hline \multicolumn{9}{|l|}{ Total lower ARI } \\
\hline Treatment failure & $44.9(41.0,48.8)$ & $66.4(64.7,68.2)$ & $23.5(21.2,26.0)$ & $84.0(82.4,85.5)$ & $24.4(21.1,27.9)$ & $83.4(82.0,84.8)$ & $25.3(21.9,28.9)$ & $82.8(81.3,84.1)$ \\
\hline Mortality & $61.2(54.7,67.4)$ & $66.2(64.5,67.8)$ & $11.7(9.97,13.6)$ & $95.9(95.0,96.7)$ & $33.8(27.8,40.2)$ & $83.1(81.8,84.4)$ & $12.8(10.3,15.7)$ & $94.5(93.6,95.3)$ \\
\hline \multicolumn{9}{|l|}{ Bronchitis } \\
\hline Treatment failure & $0.95(0.02,5.19)$ & $96.0(93.7,97.7)$ & $5.56(0.14,27.3)$ & $79.7(75.9,83.1)$ & NA & NA & NA & NA \\
\hline Mortality & $0.00(0.00,84.2)$ & $96.6(94.7,98.0)$ & $0.00(0.00,18.5)$ & $99.6(98.6,100)$ & NA & NA & NA & NA \\
\hline \multicolumn{9}{|l|}{ ECOPD } \\
\hline Treatment failure & $11.3(5.28,20.3)$ & $93.8(91.3,95.8)$ & $22.5(10.8,38.5)$ & $86.9(83.8,89.6)$ & $2.50(0.30,8.74)$ & $98.8(97.4,99.6)$ & $25.0(3.19,65.1)$ & $86.4(83.3,89.1)$ \\
\hline Mortality & $29.4(10.3,56.0)$ & $93.8(91.5,95.6)$ & $12.5(4.19,26.8)$ & $97.8(96.2,98.9)$ & $5.88(0.15,28.7)$ & $98.8(97.5,99.5)$ & $12.5(0.32,52.7)$ & $97.2(95.5,98.4)$ \\
\hline \multicolumn{9}{|l|}{ CAP } \\
\hline Treatment failure & $62.5(57.7,67.1)$ & $54.1(51.6,56.6)$ & $27.0(24.2,29.9)$ & $84.2(81.8,86.4)$ & $34.9(30.4,39.7)$ & $77.7(75.5,79.7)$ & $29.8(25.8,34.0)$ & $81.5(79.4,83.4)$ \\
\hline Mortality & $64.0(56.8,70.7)$ & $52.2(49.8,54.5)$ & $12.8(10.8,15.1)$ & $92.9(91.2,94.4)$ & $36.0(29.3,43.2)$ & $76.2(74.1,78.1)$ & $14.3(11.3,17.7)$ & $91.5(90.0,92.9)$ \\
\hline
\end{tabular}

${ }^{\mathrm{a} A R I}$, Acute respiratory tract infection; CAP, Community-acquired pneumonia; Cl, Confidence interval; ECOPD, Exacerbated chronic obstructive pulmonary disease; ED, Emergency department; ICU, Intensive care unit; NA, Not applicable due to small sample size; NPV, Negative predictive value; PC, Primary care; PCT, Procalcitonin; PPV, Positive predictive value. 
mortality, such as ICU admission or CAP-related complications. For these outcomes, PCT significantly improved clinical risk scores. In another large CAP study, done in Germany and including mostly low-risk patients, researchers found that PCT was a fair predictor of mortality and significantly improved clinical risk scores [24].

According to these studies, our findings are generally congruent. In low-risk patients (primary care) with respiratory infections, low PCT levels $<0.1 \mu \mathrm{g} / \mathrm{L}$ correctly identified patients at lower risk for treatment failure with a high NPV. In higher-risk populations (CAP, ICU), increased levels of PCT had high PPV mainly for mortality prediction. In ED patients and in patients with lower ARIs, PCT was a good predictor of treatment failure and mortality.

Nonetheless, several limitations should be considered when interpreting our results. First, we carried out a secondary analysis of a meta-analysis. It was designed to focus on a different question, that is, whether using PCT to guide initiation and duration of antibiotic treatment in patients with ARIs in primary care, ED and ICUs is safe and efficient in a broader patient population. Second, we did not investigate the effect of repeated measurements that might add supplementary prognostic information to admission levels. Third, we focused only on interventional trials as specified in the meta-analysis protocol and did not include any observational data that would potentially allow for larger sample sizes in the different subgroups and increases in the patient spectrum, as in observational studies less rigorous exclusion criteria usually apply. Fourth, we focused on adverse outcomes as specified in the original trials, but we were not able to look into some adverse outcomes, such as septic shock and respiratory failure, among others. Fifth, clinical risk scores such as CURB-65 and PSI for CAP and Acute Physiology and Chronic Health Evaluation for ICU patients were not routinely available and thus were not included in this analysis. Similarly, we were not able to obtain more detailed baseline data on all patients, including comorbidities and other risk factors, which would have allowed a more rigorous adjustment in the statistical models. Although this study provides new and clinically relevant information about the prognostic value of initial admission PCT values in different clinical setting and for different upper and lower ARIs, it remains unclear whether an improved initial prognostic assessment based on PCT level would translate into better triage decisions and outcomes in patients. Sixth, randomised controlled outcome studies need to be conducted for patients with ARIs in the ED and ICU to investigate whether real-life measurement of PCT adds useful prognostic information and thereby improves the daily clinical management and outcomes of patients.

\section{Conclusions}

This is the first large-scale study in which the prognostic value of admission PCT levels has been investigated throughout all clinical settings in the field of ARIs. PCT levels mainly provide prognostic information for risk stratification of patients in the ED setting and in patients with low to moderate ARIs. Future randomised controlled studies must address whether adding PCT to initial risk assessment can improve patient management and prognostication.

\section{Key messages}

- We found an increase in PCT levels across the clinical settings and ARI diagnoses, supporting the current practice of using specific PCT cutoff levels in different clinical settings.

- PCT levels are significantly associated with treatment failure and mortality in the overall population.

- The prognostic information of initial PCT levels in primary care and ICU patients is rather low, whereas admission PCT levels provide important prognostic information in the ED setting and in patients with low to moderate ARIs.

\section{Abbreviations}

ARI: Acute respiratory tract infection; AUC: Area under the curve; CAP: Community-acquired pneumonia; Cl: Confidence interval; ECOPD: Exacerbated chronic obstructive pulmonary disease; ED: Emergency department; ICU: Intensive care unit; IQR: Interquartile range; OR: Odds ratio; PCT: Procalcitonin; PSI: Pneumonia Severity Index; ROC: Receiver operating characteristic; RTI: Respiratory tract infection; VAP: Ventilator-associated pneumonia.

\section{Competing interests}

No commercial sponsor had any involvement in the design or conduct of this study, namely, the collection, management, analysis and interpretation of the data; and the preparation, decision to submit, review, or approval of the manuscript. AK, SS, PS, MC-C and BM received support from $B \cdot R \cdot A \cdot H \cdot M \cdot S$ to attend meetings and fulfil speaking engagements. PS, MC-C and BM received support from bioMérieux to attend meetings and fulfil speaking engagements. BM has served as a consultant to and received research support from B.R.A.H.M.S and bioMérieux. MB and HCB report receiving grants from $B \cdot R \cdot A \cdot H \cdot M \cdot S$ during the conduct of the study. DS and MT received research support from B.R.A.H.M.S. TW reports receiving personal fees from Thermo Fisher and Becton Dickinson and grants from Siemens Diagnostics during the conduct of the study. Outside the presently reported study, TW reports receiving personal fees from Bayer, Novartis, Basilea Pharmaceutica, Pfizer, Cubist, AstraZeneca, GSK and MSD. C-EL reports receiving personal fees from bioMérieux, grants from Janssen and grants from Bayer outside the submitted work. MW reports receiving personal fees from Pfizer and Gilead outside the submitted work. FT reports receiving grants from Abbott, AstraZeneca, Pfizer and Schering-Plough outside the submitted work. All other authors declare that they have no competing interests.

\section{Authors' contributions}

All authors made substantive intellectual contributions to this study. AK, BM and PS conducted statistical analyses and drafted the first manuscript. MB, MC-C, DS, LB, MW, KBB, LW, OB, TW, SS, VN, MT, HB, C-EL, JC and FT were in charge of the acquisition of patient data in the included trials, provided individual patient data from their respective trials and resolved queries about 
their trial data as needed. For this article, they made substantial contributions to conception and design and took an active part in acquisition, analysis and interpretation of data. NB is a librarian who did the search for our metaanalysis and helped in the search and interpretation of studies. She gave feedback on the manuscript and was part of the team that developed the ideas for the meta-analysis. All authors contributed to the interpretation of data and to the revising of the manuscript critically for important intellectual content. All authors approved the final version of the manuscript and agreed to be accountable for all aspects of the work in ensuring that questions related to the accuracy or integrity of any part of the work are appropriately investigated and resolved.

\section{Acknowledgements}

We thank Blandine Pasquet (Institut national de la santé et de la recherche médicale (INSERM), Paris) for assisting with data queries. We thank Qing Wang, PhD (Basel Institute for Clinical Epidemiology and Biostatistics, University Hospital Basel, Switzerland), for her help with the translation of an article published in Chinese. We thank Dr Jeffrey Greenwald (Massachusetts General Hospital (MGH), Boston, MA, USA) for scientific discussion. None of these persons received any compensation for their help with this study.

\section{Author details}

${ }^{1}$ University Department of Medicine, Kantonsspital Aarau, Tellstrasse, 5001 Aarau, Switzerland. ${ }^{2}$ Basel Institute for Clinical Epidemiology and Biostatistics, University Hospital Basel, Hebelstrasse 10, Basel 4031, Switzerland. ${ }^{3}$ Division of Endocrinology, Diabetology and Clinical Nutrition, University Hospital Basel, Hebelstrasse 10, Basel 4031, Switzerland. ${ }^{4}$ Clinic of Pneumology and Pulmonary Cell Research, University Hospital Basel, Hebelstrasse 10, Basel 4031, Switzerland. ${ }^{5}$ Service de Réanimation Médicale, Université Paris 7-Denis Diderot, Hôpital Bichat-Claude-Bernard, Assistance Publique-Hôpitaux de Paris (AP-HP), Henri Huchard Paris Cedex 18, Paris 75877, France. ${ }^{6}$ Department of Infectious Diseases, Aarhus University Hospital, Skejby, Palle Juul-Jensens Boulevard 99, 8200 Aarhus N, Denmark. Department of Internal and Geriatric Medicine, Shanghai Jiao Tong University Affiliated Sixth People's Hospital, Huan Hu Xi San Road, Pudong New Area, Shanghai 201306, China. ${ }^{8}$ Department of Pulmonary Medicine, Hannover Medical School, Carl-Neuberg-Str. 1, Hannover 30659, Germany. ${ }^{9}$ Department of Anesthesiology and Intensive Care Medicine, Düren Hospital, Roonstraße 30, Düren 52351, Germany. ${ }^{10}$ Intensive Care, Universidade Federal de Minas Gerais, 6627 - Pampulha, Belo Horizonte - MG 31270-901, Brazil. ${ }^{11}$ Department of Clinical Epidemiology and Biostatistics, McMaster University, 1280 Main Street West, Hamilton, Ontario L8S4L8, Canada. ${ }^{12}$ Service de Réanimation Médicale, Université Paris 6-Pierre et Marie Curie, Hôpital PitiéSalpêtrière, AP-HP, 47-83 boulevard de I'Hôpital, Paris 75651, France. ${ }^{13}$ AP-HP, Hôpitaux Universitaires Paris Nord Val de Seine, Département d'Epidémiologie Biostatistique et Recherche Clinique, Université Paris Diderot, Sorbonne Paris Cité, UMR 738, INSERM, UMR 738, INSERM, CIE801, 5 Rue Thomas Mann, Paris, Cedex 13 75013, France.

\section{Received: 14 September 2014 Accepted: 6 February 2015} Published online: 06 March 2015

\section{References}

1. Warren HS. Strategies for the treatment of sepsis. N Engl J Med. 1997;336:952-3.

2. Parrillo JE, Parker MM, Natanson C, Suffredini AF, Danner RL, Cunnion RE, et al. Septic shock in humans: advances in the understanding of pathogenesis, cardiovascular dysfunction, and therapy. Ann Intern Med. 1990;113:227-42.

3. Kumar A, Roberts D, Wood KE, Light B, Parrillo JE, Sharma S, et al. Duration of hypotension before initiation of effective antimicrobial therapy is the critical determinant of survival in human septic shock. Crit Care Med. 2006;34:1589-96.

4. Masson S, Caironi P, Spanuth E, Thomae R, Panigada M, Sangiorgi G, et al. Presepsin (soluble CD14 subtype) and procalcitonin levels for mortality prediction in sepsis: data from the Albumin Italian Outcome Sepsis trial. Crit Care. 2014;18:R6

5. Garnacho-Montero J, Huici-Moreno MJ, Gutiérrez-Pizarraya A, López I, Márquez-Vácaro JA, Macher $H$, et al. Prognostic and diagnostic value of eosinopenia, C-reactive protein, procalcitonin, and circulating cell-free DNA in critically ill patients admitted with suspicion of sepsis. Crit Care. 2014;18:R116.

6. Suberviola B, Castellanos-Ortega A, Ruiz Ruiz A, Lopez-Hoyos M, Santibañez M. Hospital mortality prognostication in sepsis using the new biomarkers suPAR and proADM in a single determination on ICU admission. Intensive Care Med. 2013;39:1945-52.

7. Schuetz P, Litke A, Albrich WC, Mueller B. Blood biomarkers for personalized treatment and patient management decisions in community-acquired pneumonia. Curr Opin Infect Dis. 2013;26:159-67.

8. Magrini L, Gagliano G, Travaglino F, Vetrone F, Marino R, Cardelli P, et al. Comparison between white blood cell count, procalcitonin and $C$ reactive protein as diagnostic and prognostic biomarkers of infection or sepsis in patients presenting to emergency department. Clin Chem Lab Med. 2014;52:1465-72.

9. Li Z, Wang H, Liu J, Chen B, Li G. Serum soluble triggering receptor expressed on myeloid cells-1 and procalcitonin can reflect sepsis severity and predict prognosis: a prospective cohort study. Mediators Inflamm. 2014;2014:641039.

10. Zhao Y, Li C, Jia Y. Evaluation of the Mortality in Emergency Department Sepsis score combined with procalcitonin in septic patients. Am J Emerg Med. 2013:31:1086-91.

11. Pierrakos C, Vincent JL. Sepsis biomarkers: a review. Crit Care. 2010;14:R15.

12. Karlsson $S$, Heikkinen $M$, Pettilä $V$, Alila S, Väisänen S, Pulkki $K$, et al. the Finnsepsis Study Group. Predictive value of procalcitonin decrease in patients with severe sepsis: a prospective observational study. Crit Care. 2010;14:R205.

13. Georgopoulou AP, Savva A, Giamarellos-Bourboulis EJ, Georgitsi M, Raftogiannis M, Antonakos N, et al. Early changes of procalcitonin may advise about prognosis and appropriateness of antimicrobial therapy in sepsis. J Crit Care. 2011;26:331. e1-e7.

14. Haasper C, Kalmbach M, Dikos GD, Meller R, Müller C, Krettek C, et al. Prognostic value of procalcitonin (PCT) and/or interleukin-6 (IL-6) plasma levels after multiple trauma for the development of multi organ dysfunction syndrome (MODS) or sepsis. Technol Health Care. 2010;18:89-100.

15. Schuetz P, Briel M, Christ-Crain M, Stolz D, Bouadma L, Wolff M, et al. Procalcitonin to guide initiation and duration of antibiotic treatment in acute respiratory infections: an individual patient data meta-analysis. Clin Infect Dis. 2012;55:651-62.

16. Schuetz P, Müller B, Christ-Crain M, Stolz D, Tamm M, Bouadma L, et al. Procalcitonin to initiate or discontinue antibiotics in acute respiratory tract infections. Cochrane Database Syst Rev. 2012;9:CD007498. doi:10.1002/ 14651858.CD007498.pub2.

17. Mandell LA, Wunderink RG, Anzueto A, Bartlett JG, Campbell GD, Dean NC, et al. Infectious Diseases Society of America/American Thoracic Society consensus guidelines on the management of community-acquired pneumonia in adults. Clin Infect Dis. 2007;44:S27-72.

18. Schuetz P, Aujesky D, Muller C, Muller B. Biomarker-guided personalised emergency medicine for all - hope for another hype? Swiss Med Wkly. 2015;145:w14079.

19. Niederman MS, Mandell LA, Anzueto A, Bass JB, Broughton WA, Campbell $\mathrm{GD}$, et al. Guidelines for the management of adults with communityacquired pneumonia: diagnosis, assessment of severity, antimicrobial therapy, and prevention. Am J Respir Crit Care Med. 2001;163:1730-54

20. Woodhead M, Blasi F, Ewig S, Huchon G, Leven M, Ortqvist A, et al. Guidelines for the management of adult lower respiratory tract infections. Eur Respir J. 2005;26:1138-80.

21. Schuetz P, Koller M, Christ-Crain M, Steyerberg E, Stolz D, Müller C, et al. Predicting mortality with pneumonia severity scores: importance of model recalibration to local settings. Epidemiol Infect. 2008;136:1628-37.

22. Huang D, Weissfeld L, Kellum J, Yealy D, Kong L, Martino M, et al. Risk prediction with procalcitonin and clinical rules in community-acquired pneumonia. Ann Emerg Med. 2008;52:48-58. e2.

23. Schuetz P, Suter-Widmer I, Chaudri A, Christ-Crain M, Zimmerli W, Mueller B, et al. Prognostic value of procalcitonin in community-acquired pneumonia. Eur Respir J. 2011;37:384-92.

24. Krüger S, Ewig S, Kunde J, Hartmann O, Marre R, Suttorp N, et al. the CAPNETZ study group. Assessment of inflammatory markers in patients with community-acquired pneumonia-influence of antimicrobial pre-treatment: results from the German competence network CAPNETZ. Clin Chim Acta. 2010;411:1929-34. 